Article

\title{
Early-Life Intervention Using Fecal Microbiota Combined with Probiotics Promotes Gut Microbiota Maturation, Regulates Immune System Development, and Alleviates Weaning Stress in Piglets
}

\author{
Quanhang Xiang ${ }^{1}{ }^{\oplus}$, Xiaoyu Wu ${ }^{1}$, Ye Pan ${ }^{1}$, Liu Wang ${ }^{1}$, Chenbin Cui ${ }^{1}$, Yuwei Guo ${ }^{1}$, \\ Lingling Zhu ${ }^{1}$, Jian Peng ${ }^{1,2,3, *}$ and Hongkui Wei ${ }^{1,2,3, *}$ \\ 1 Department of Animal Nutrition and Feed Science, College of Animal Science and Technology, Huazhong \\ Agricultural University, Wuhan 430070, China; xiangquanhang@webmail.hzau.edu.cn (Q.X.); \\ 13586414898@163.com (X.W.); panye19910504@hotmail.com (Y.P.); wang101@sbtjt.com (L.W.); \\ cuichenbin@webmail.hzau.edu.cn (C.C.); gywmizu@163.com (Y.G.); hul66052668@163.com (L.Z.) \\ 2 The Cooperative Innovation Center for Sustainable Pig Production, Wuhan 400700, China \\ 3 Hubei Agricultural Sciences and Technology Innovation Center, Wuhan 430070, China \\ * Correspondence: pengjian@mail.hzau.edu.cn (J.P.); weihongkui@mail.hzau.edu.cn (H.W.)
}

Received: 16 December 2019; Accepted: 7 January 2020; Published: 13 January 2020

check for updates

\begin{abstract}
Previous studies have suggested that immune system development and weaning stress are closely related to the maturation of gut microbiota. The early-life period is a "window of opportunity" for microbial colonization, which potentially has a critical impact on the development of the immune system. Fecal microbiota transplantation (FMT) and probiotics are often used to regulate gut microbial colonization. This study aims to test whether early intervention with FMT using fecal microbiota from gestation sows combined with Clostridium butyricum and Saccharomyces boulardii (FMT-CS) administration could promote the maturation of gut microbiota and development of immune system in piglets. Piglets were assigned to control $(n=84)$ and FMT-CS treatment $(n=106)$, which were treated with placebo and bacterial suspension during the first three days after birth, respectively. By $16 \mathrm{~S}$ rRNA gene sequencing, we found that FMT-CS increased the $\alpha$-diversity and reduced the unweighted UniFrac distances of the OTU community. Besides, FMT-CS increased the relative abundance of beneficial bacteria, while decreasing that of opportunistic pathogens. FMT-CS also enhanced the relative abundance of genes related to cofactors and vitamin, energy, and amino acid metabolisms during the early-life period. ELISA analysis revealed that FMT-CS gave rise to the plasma concentrations of IL-23, IL-17, and IL-22, as well as the plasma levels of anti-M.hyo and anti-PCV2 antibodies. Furthermore, the FMT-CS-treated piglets showed decreases in inflammation levels and oxidative stress injury, and improvement of intestinal barrier function after weaning as well. Taken together, our results suggest that early-life intervention with FMT-CS could promote the development of innate and adaptive immune system and vaccine efficacy, and subsequently alleviate weaning stress through promoting the maturation of gut microbiota in piglets.
\end{abstract}

Keywords: early-life intervention; fecal microbiota transplantation; gut microbiota; immune system; weaning stress; piglet

\section{Introduction}

The immune system of newborn animals is generally considered to be immature, which is closely related to a series of early diseases [1-4]. For instance, the immaturity of the intestinal immune system is regarded as one of the most important reasons for early-life diarrhea and early-weaning stress in 
mammals [5], and it has been confirmed that the development and maturation of the mammal intestinal immune system occur in infancy, even in the fetal period [6-8]. Hence, promoting the maturation of the intestinal immune system in the early-life period is of great significance for the improvement of growth, development, and disease resistance of neonatal mammals.

Gut microbiota plays a vital role in the development and maturation of the intestinal immune system [9-11]. Previous research has indicated that colonization history and donor-recipient compatibility play important roles in microbial colonization and community of neonatal mammals [12-14]. There has been increasing evidence suggesting that early intervention of the gut microbiota during the critical "window of opportunity" period may be a promising method to improve intestinal microbial colonization [15-18]. Newborn piglets are usually used as an ideal animal model for studying human nutrition and physiology [19]. In our previous study, early intervention with mature maternal fecal microbiota transplantation (FMT) was found to have positive effects on the improvement of growth performance and immunity and decrease diarrhea in the newborn piglet model [20]. However, the developmental changes in the intestinal microbiota after early intervention remain elusive.

Moreover, aerobic and facultative anaerobic bacteria contribute to the consumption of oxygen in the intestine, which can promote the colonization of strict anaerobes [21,22]. Saccharomyces boulardii (S. boulardii) is an aerobic yeast [23], and early intervention with S. boulardii can alleviate porcine neonatal diarrhea $[24,25]$. Clostridium butyricum (C. butyricum) is a probiotic and has been used for the clinical treatment of premature infants with diarrhea by promoting the maturation of the immune function [26-28].

Therefore, we hypothesize that the modulation of gut microbiota by mature maternal FMT combined with C. butyricum and S. boulardii (FMT-CS) oral administration during the early-life period would improve host immune system development and alleviate weaning stress. By using a newborn piglet model, this study might provide novel insights into the improvement of the immune system development in neonatal mammals.

\section{Results}

\subsection{Bacterial Alpha-Diversity and Beta-Diversity}

The fecal microbiota of piglets in the control and treatment groups at the age of 7, 27, 35, and $56 \mathrm{~d}$ was analyzed by sequencing the bacterial $16 \mathrm{~S}$ rRNA V3-V4 region. Early-life intervention with FMT-CS significantly increased the Chao1 estimator and ACE estimator of fecal microbiota at the age of $7 \mathrm{~d}$ $(p<0.05)$ and $27 \mathrm{~d}(p<0.01)$ in comparison with those in control piglets, and showed a trend to increase the Shannon diversity index at the age of $27 \mathrm{~d}(0.05<p<0.1)$. There was no significant difference in the Simpson index (Figure 1A). The NMDS plot of the dissimilarity of microbial community also revealed a distinct microbial community structure between the control and treatment groups (Figure 1B), and the ANOSIM for differences between the two groups was significant at the age of $7 \mathrm{~d}(\mathrm{R}=0.7868$, $p=0.001), 27 \mathrm{~d}(\mathrm{R}=0.2044, p=0.004)$, and $35 \mathrm{~d}(\mathrm{R}=0.3578, p=0.001)$. However, there was no significant difference in microbial community at the age of $56 \mathrm{~d}$ (ANOSIM: $\mathrm{R}=0.0283, p=0.251$ ). As shown in Figure 1C, the control piglets showed a higher variability of the OTU community than the treated piglets. Moreover, the fecal microbial community structure at the age of $7 \mathrm{~d}$ was more similar to that at the age of $56 \mathrm{~d}$ in the treated piglets than in the control piglets. 
A

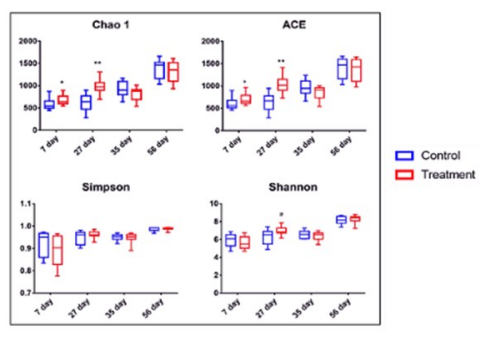

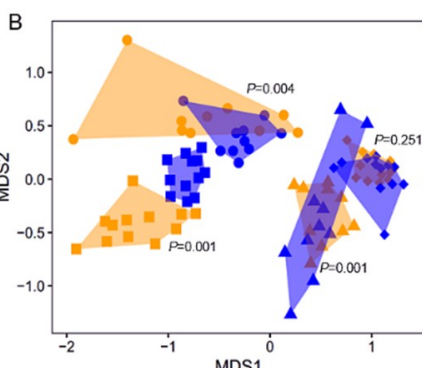

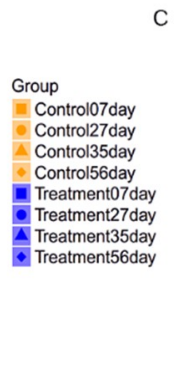

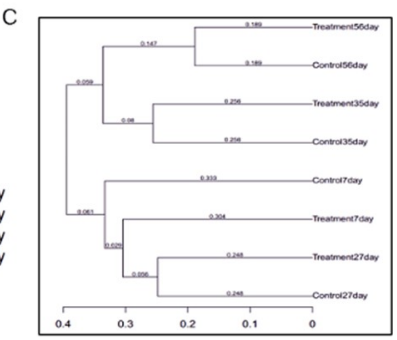

Figure 1. $\alpha$ - and $\beta$-diversity of fecal microbiota in piglets after early intervention with FMT-CS. (A) Chao1 estimator, ACE estimator, Simpson index, and Shannon diversity index between control group and treatment group. (B) NMDS analysis of the fecal microbiota structure between the control groups and treatment groups. (C) $\beta$-diversity based on the unweighted UniFrac distances of the OTU community.

\subsection{Early-Life Intervention with FMT-CS Affected the Composition of Fecal Microbiota}

The microbiota composition of the fecal samples of piglets at the age of $7 \mathrm{~d}, 27 \mathrm{~d}, 35 \mathrm{~d}$, and $56 \mathrm{~d}$ were assessed by deep sequencing of the V3-V4 region of the $16 \mathrm{~S}$ rRNA genes. The relative abundance of the fecal microbiota at the phylum and genus levels is displayed in Figure 2. At the phylum level (Figure 2A), Firmicutes and Bacteroidetes were the most dominant phyla in both control and treated piglets. In treated piglets, the third most dominant phylum was Spirochaetes before weaning, and was Actinobacteria after weaning. Notably, a rapid increase in the relative abundance of Actinobacteria might be a signal of weaning stress. In control piglets, Fusobacteria and Proteobacteria were the third and fourth most dominant phyla before weaning ( $7 \mathrm{~d}$ and $27 \mathrm{~d}$ ); while after weaning ( $35 \mathrm{~d}$ and $56 \mathrm{~d}$ ), the relative abundance of Fusobacteria drastically decreased, and Actinobacteria became the third most dominant phylum. At the genus level (Figure 2B), Prevotella and Lactobacillus were dominant in both control and treated piglets. In control piglets, the other two major genera were Fusobacterium and Bacteroides at the age of $7 \mathrm{~d}$ and $27 \mathrm{~d}$, Collinsella and Catenibacterium at the age of $35 \mathrm{~d}$, and Roseburia and Faecalibacterium at the age of $56 \mathrm{~d}$; while in treated piglets, the other two major genera were Treponema and $p$-75-a5, Treponema and Phascolarctobacterium, Collinsella and Bulleidia, and Faecalibacterium and Roseburia at the age of $7 \mathrm{~d}, 27 \mathrm{~d}, 35 \mathrm{~d}$, and $56 \mathrm{~d}$, respectively.
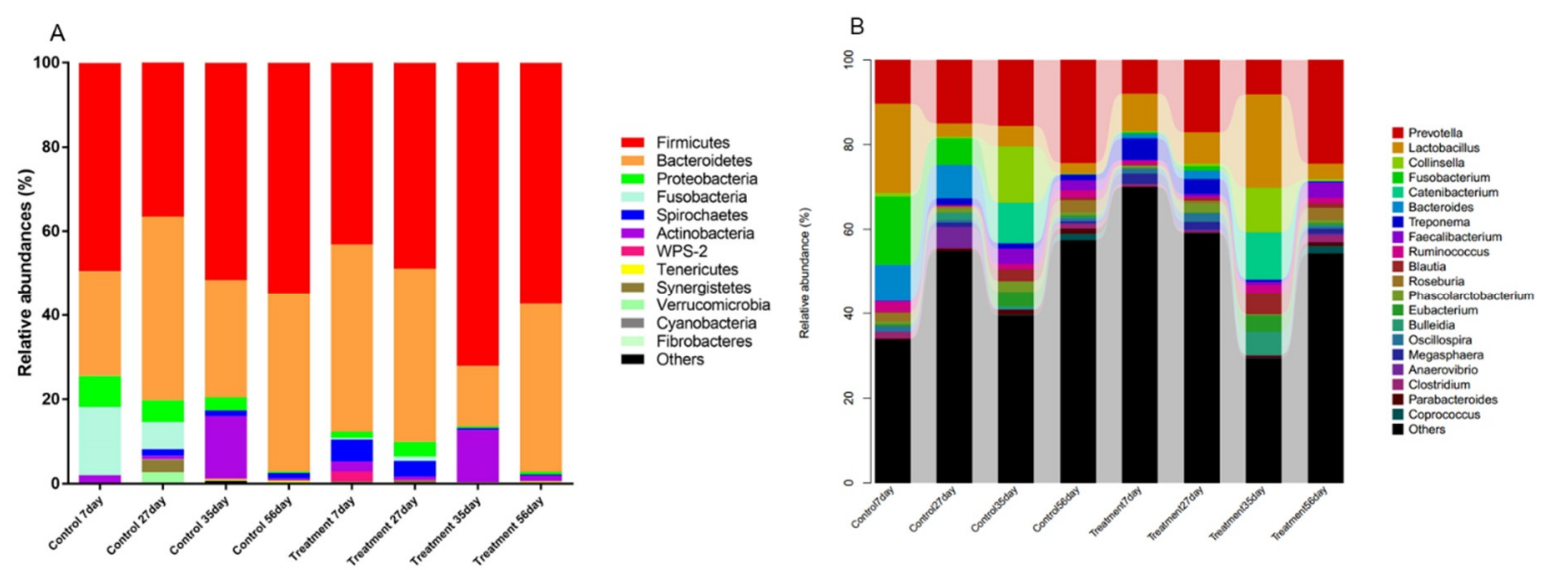

Figure 2. Microbiota composition determined by $16 \mathrm{~S}$ rRNA gene sequencing of fecal samples. The relative abundance of the phyla (A) and genera (B) of fecal bacteria present in suckling piglets of both the control and treatment groups.

\subsection{Early-Life Intervention with FMT-CS Affected the Organism-Level Microbiome Phenotypes}

In order to further understand the structure of gut microbiota, BugBase analysis, for analyzing the phenotype of microbiome samples, was performed to predict the proportions of biofilm forming, 
pathogenic, mobile element containing, oxygen utilizing, and oxidative stress tolerant microorganisms (Figure 3). In both the control and treated piglets, the relative abundance of aerobes gradually decreased while that of anaerobes increased with time. There was a slight decrease of aerobes in treated piglets at the age of $7 \mathrm{~d}$ compared with in the control piglets (pairwise Mann-Whitney-Wilcoxon test $p$-value $\left(p_{1}\right)=0.1431$; FDR-corrected pairwise $p$-value $\left.\left(p_{2}\right)=0.1822\right)$. At the age of $35 \mathrm{~d}$, significant increases in aerobes $\left(p_{1}=0.0028, p_{2}=0.0073\right)$ and decreases in anaerobes $\left(p_{1}=0.0355, p_{2}=0.0717\right)$ were observed in treated piglets. Notably, significant decreases in potential pathogenic microorganisms (at $35 \mathrm{~d}$; $\left.p_{1}=0.0007, p_{2}=0.0029\right)$ and mobile element containing microorganisms (at $7 \mathrm{~d} ; p_{1}=0.0003, p_{2}=0.0005$ ) were found in treated piglets.
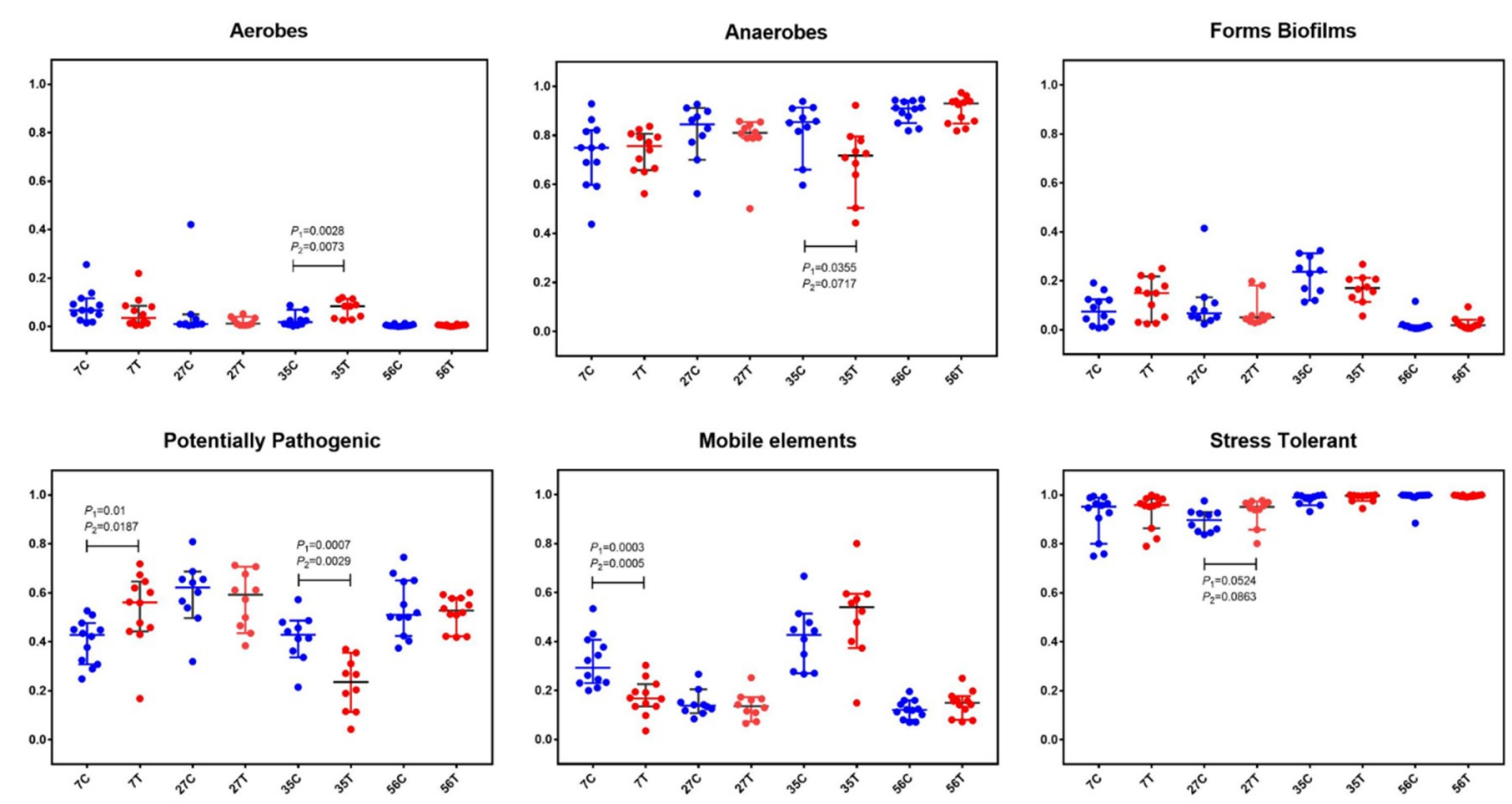

Figure 3. BugBase analysis based on the $16 \mathrm{~S}$ rRNA gene sequencing dataset. The outcome was grouped according the modules ( $x$-axis). Blue dots represent the control group and red dots represent the treatment group. The relative abundance is presented on the $y$-axis. Pairwise Mann-Whitney-Wilcoxon tests $\left(p_{1}\right)$ and FDR-corrected pairwise tests $\left(p_{2}\right)$ were performed for data analysis.

\subsection{Early-Life Intervention with FMT-CS Affected the Dominant Bacteria}

Linear discriminant analysis (LDA) effect size (LefSe) analysis was performed to confirm the effects of early-life intervention with FMT-CS on the fecal microbiota of piglets at $7 d, 27 d, 35 d$, and $56 \mathrm{~d}$ (Figure 4). The results revealed that at the age of $7 \mathrm{~d}$, Fusobacteria, Proteobacteria, Bacilli, and Verrucomicrobiae were enriched in control piglets, while Bacteroidetes, Synergistetes, Spirochaetes, Erysipelotrichi and Verruco-5 were dominant in treated piglets (Figure 4A). At the age of $27 \mathrm{~d}$, Clostridiales, Coriobacteriales, and Lactobacillales were significantly increased in the treatment group $(p<0.05)$, while a significant increase in Aeromonadales was observed in the control group $(p<0.05)$. At the genus level, 10 known genera (Collonsella, Enterococcus, Blautia, Coprococcus, Dorea, rc4-4, Faecalibacterium, Oscillospira, Ruminococcaceae, and $p-75-a 5)$ were significantly increased at $27 \mathrm{~d}$ in treated piglets $(p<0.05)$ (Figure 4B). At the age of $35 \mathrm{~d}$, Lactobacillales, Bacillales, and Sphingomonadales were significantly increased in the treatment group $(p<0.05)$, while Bacteroidales and Clostridiales showed dramatic increases in the control group. At the genus level, three genera (Lactobacillus, Rummeliibacillus, and Sphingobium) were significantly increased in the treatment group and Phascolarctobacterium showed a significant increase in the control group $(p<0.05)$ (Figure $4 \mathrm{C})$. At the age of $56 \mathrm{~d}$, Actinobacteria and Christensenellaceae were enriched in control piglets; while the treated piglets showed enrichment of Gammaproteobacteria and Blautia. Notably, there were obvious increases in some harmful bacteria (Fusobacterium, Akkermansia, and Enterobacteriacease) in control piglets (Figure 4D). 
A

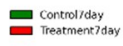

Cladogram

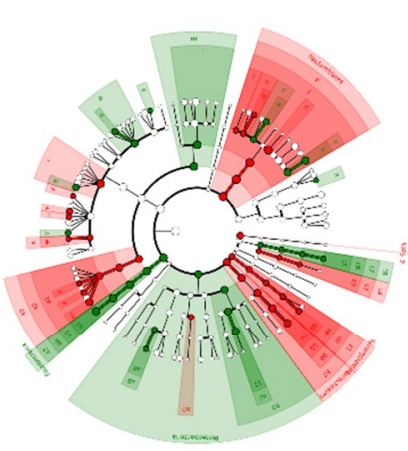

C

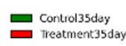

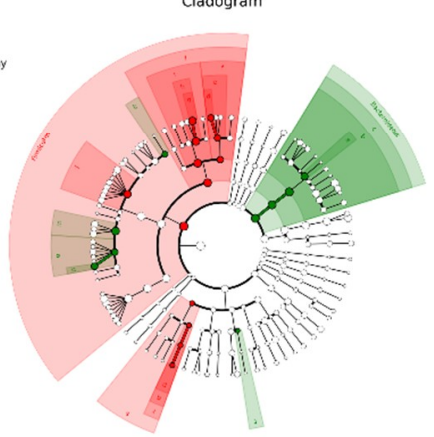

B
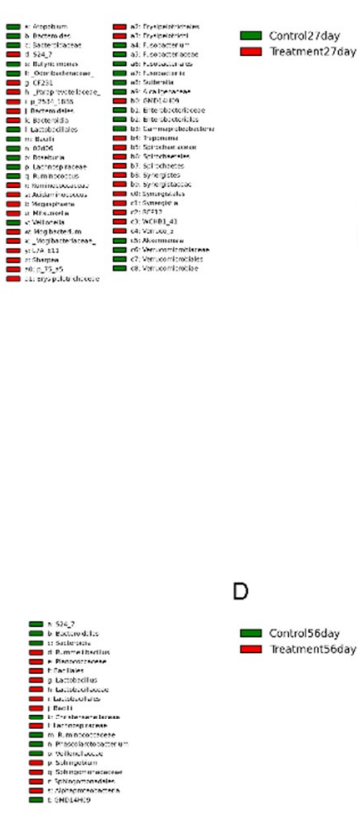

D

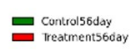

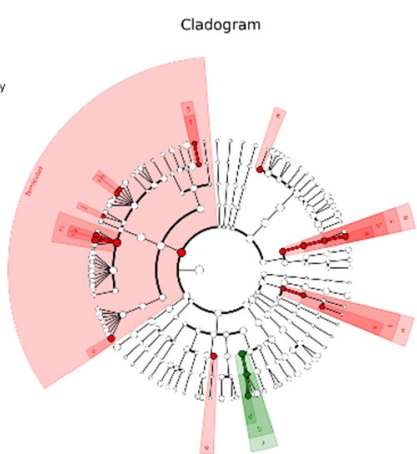

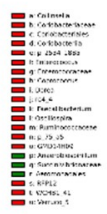

Cladogram

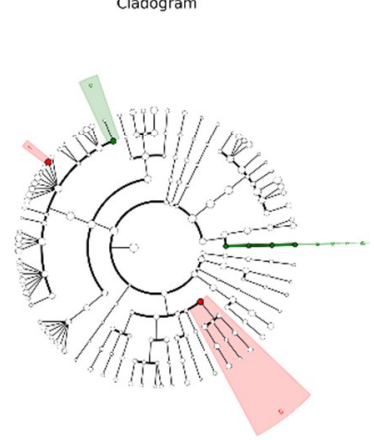

Figure 4. Differential enrichment of fecal microbiota. LefSe analysis of fecal microbiota at the age of 7 $\mathrm{d}(\mathbf{A}), 27 \mathrm{~d}(\mathbf{B}), 35 \mathrm{~d}(\mathbf{C})$, and D 56 (D) after early intervention with FMT-CS. The cladogram shows the microbial species with significant differences between groups in LEfSe analysis. Red indicates the treatment group and green indicates the control group.

\subsection{Early-Life Intervention with FMT-CS Affected the Changing Pattern of Differential Bacteria}

To further analyze the effect of early-life intervention with FMT-CS on the composition of bacteria, we heatmap-analyzed the differential bacteria at the phylum and genus levels based on the results of the metastats analysis (Figure 5), and the relative abundance of differential bacteria is shown in Supplemental Tables S1 and S2. At the phylum level (Figure 5A), five groups of differential bacteria were clustered in the dendrogram. Early-life intervention with FMT-CS increased the relative proportion of cluster A and C, which included more dominant bacteria such as Firmicutes and Bacteroidetes. In the control group, the relative proportion of harmful bacteria (such as Fusobacteria and Proteobacteria) was higher, especially in cluster D. At the genus level (Figure 5B), there were seven major clusters, with five sub-clusters for cluster 6 . In the treatment group, beneficial bacteria were enriched in the early life of piglets (cluster 3, 7) and post-weaning period (cluster 1). However, the relative proportion of opportunistic pathogens (such as Fusobacterium in cluster 2) was high in control piglets. In addition, cluster S1 and S2 contained the two most dominant bacteria, Prevotella and Lactobacillus, which were enriched in treated piglets at $35 \mathrm{~d}$. 

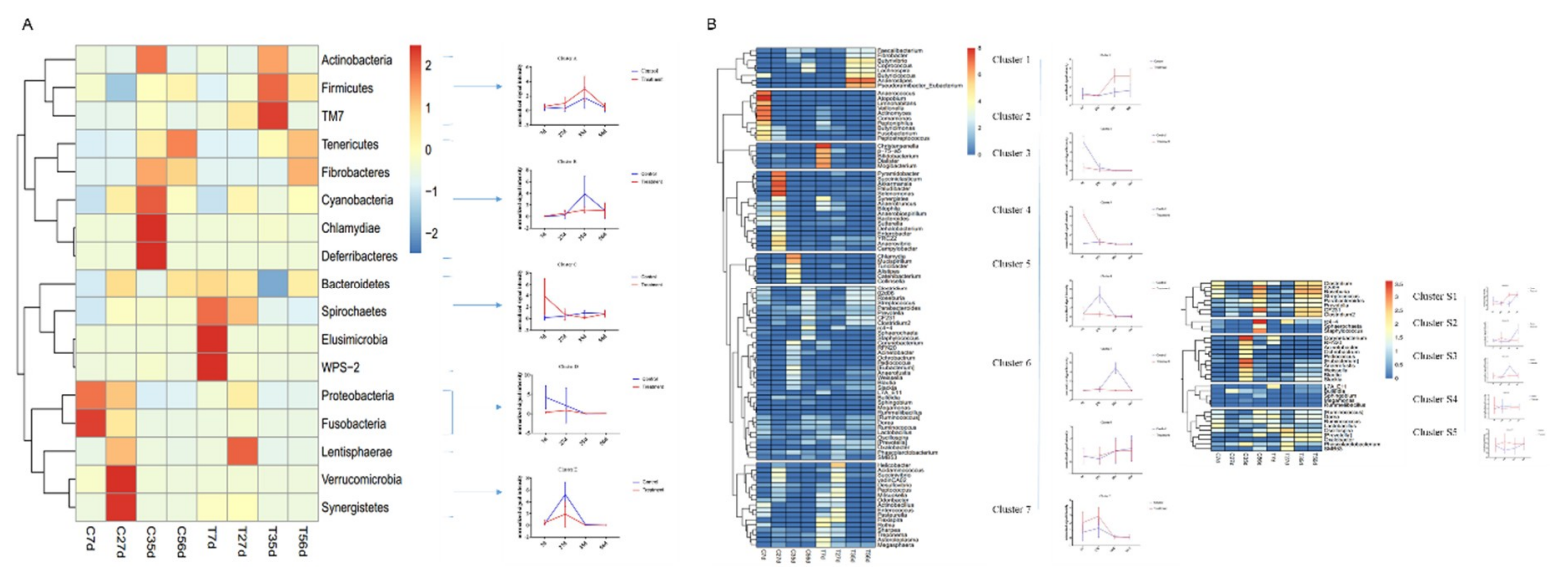

Figure 5. Hierarchical clustering and heatmap of the differential genes at the phylum level (A) and genus level (B) between groups at different time based on the results of the metastats analysis.

\subsection{Comparison of Metabolic Pathway Abundance}

We predicted the microbial metagenome with 16S rRNA gene sequencing using phylogenetic investigation of communities by reconstruction of unobserved states (PICRUSt) [29]. The results showed that the genes that regulate the metabolism of vitamins, energy, and glycan, and are highly expressed in adult pigs [30,31], had significantly higher expression in treated piglets. Besides, genes that regulate amino acid metabolism, biosynthesis of other secondary metabolites, metabolism of terpenoids and polyketides, and nucleotide metabolism were more abundant in the treatment group than in the control group at an age of $7 \mathrm{~d}$. However, no significant differences were found for these genes between groups at the age of $27 \mathrm{~d}, 35 \mathrm{~d}$, and $56 \mathrm{~d}$ (Figure 6). These results indicated that the microbiota of the treated piglets was more mature than that of the control piglets.
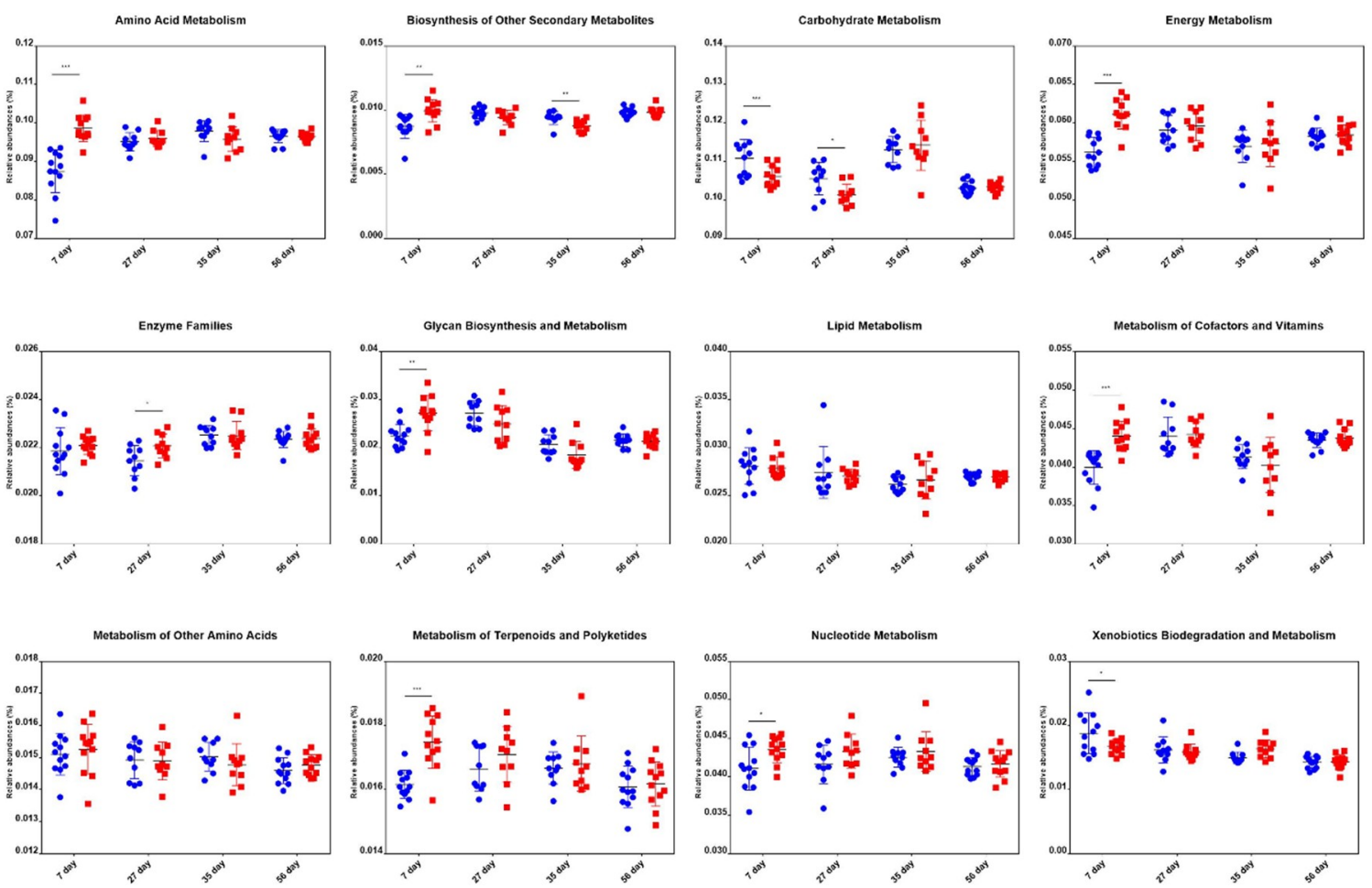

Figure 6. Differences in metabolic functional genes in piglets of the control and treatment groups at the age of $7 \mathrm{~d}, 27 \mathrm{~d}, 35 \mathrm{~d}$, and $56 \mathrm{~d}$. Blue dots and red dots represent the control and treatment groups, respectively. ${ }^{*} p<0.05,{ }^{* *} p<0.01,{ }^{* * *} p<0.001$. 


\subsection{Early-Life Intervention with FMT-CS Affected Cytokines of the Innate Immune System}

In order to evaluate the effect of early-life intervention with FMT-CS on the innate immune system, the plasma cytokines were determined and compared. As shown in Figure 7, at the age of $7 \mathrm{~d}$, plasma IL-23 was significantly increased in treatment piglets compared with in control piglets, while IL-1 $\beta$ was significantly decreased (Figure 7A). In addition, plasma IL-17 and IL-22 were increased in treated piglets as well (Figure 7B). The concentrations of plasma IL-17, IL-22, and INF- $\gamma$ consistently increased after birth, and reached the maximum at the age of $35 \mathrm{~d}$ (Figure 7C-E). Moreover, the concentrations of plasma IL-17, IL-22, and INF- $\gamma$ of treated piglets were generally higher than those in control piglets.
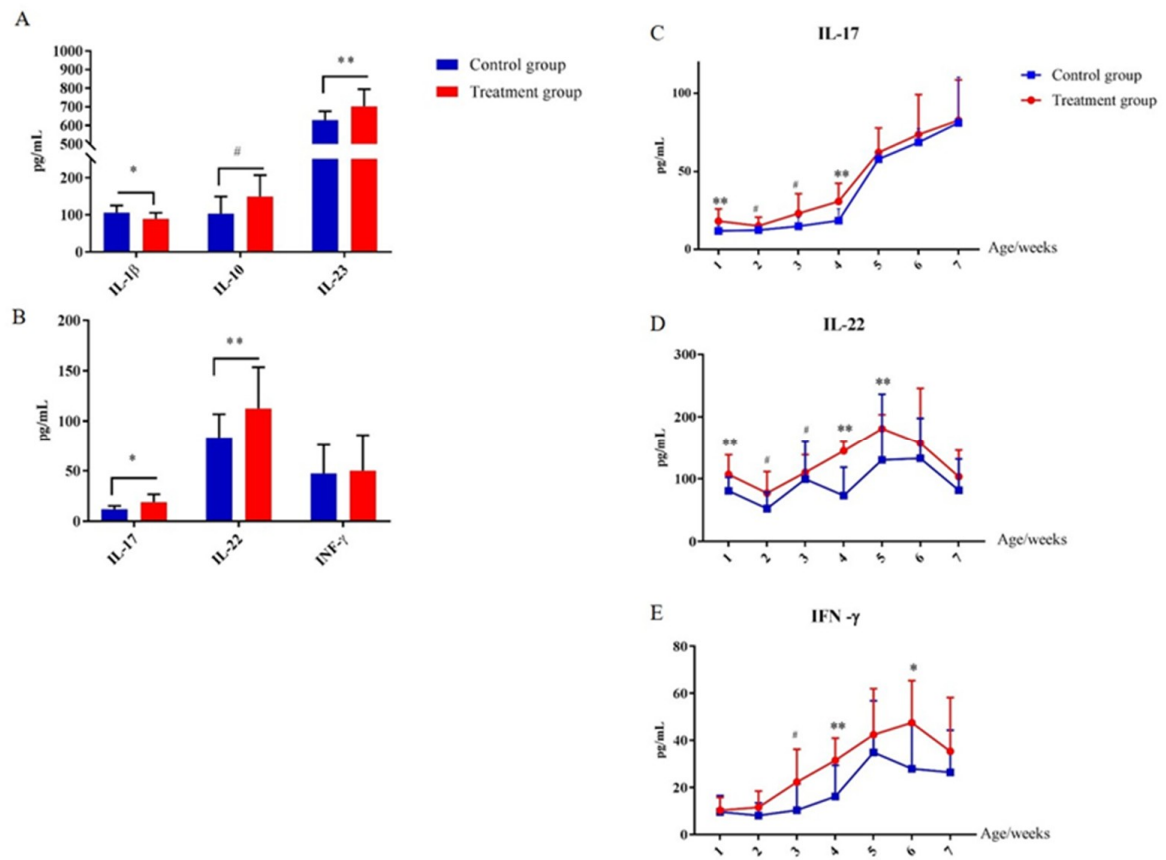

Figure 7. Effect of early-life intervention with FMT-CS on the development of type 3 innate lymphoid cells. (A) Cytokines (IL-1 $\beta$, IL-10, and IL-23) associated with regulation of ILC3, and (B) cytokines (IL-17, IL-22, and IFN- $\gamma$ ) secreted by ILC3s in the plasma of piglets at the age of $7 \mathrm{~d}$. (C-E) Plasma concentrations of IL-17, IL-22, and IFN- $\gamma$ at indicated time points after birth. Values are expressed as mean \pm SEM. ${ }^{\#} 0.05<p<0.1,{ }^{*} p<0.05,{ }^{* *} p<0.01$.

\subsection{Early-Life Intervention with FMT-CS Affected the Adaptive Immune Homeostasis}

The effects of early gut microbiota intervention on the plasma levels of IgG, IgM, and fecal sIgA are presented in Table 1. Compared with control piglets, the treated piglets showed significant increases in the plasma concentrations of $\operatorname{IgG}, \operatorname{IgM}$, and fecal $\operatorname{sgA}$ at 14, 21, 35, and $56 \mathrm{~d}(p<0.05)$.

Table 1. Effects of early intervention with FMT-CS on fecal sIgA, plasma IgG, and IgM concentrations of piglets.

\begin{tabular}{ccccc}
\hline Item & Control & Treatment & SEM & $p$-Value \\
\hline Day 14 & & & & \\
Fecal sIgA, $\mu \mathrm{g} / \mathrm{g}$ & $128.24^{\mathrm{b}}$ & $168.53^{\mathrm{a}}$ & 1.35 & $<0.0001$ \\
Plasma IgG, mg/mL & $19.8^{\mathrm{b}}$ & $26.94^{\mathrm{a}}$ & 1.47 & 0.0135 \\
Plasma IgM, mg/mL & $15.48^{\mathrm{b}}$ & $22.46^{\mathrm{a}}$ & 1.1 & $<0.0001$ \\
Day 21 & & & & \\
Fecal sIgA, $\mu \mathrm{g} / \mathrm{g}$ & $114.19^{\mathrm{b}}$ & $145.92^{\mathrm{a}}$ & 1.8 & 0.0242 \\
Plasma IgG, mg/mL & $20.56^{\mathrm{b}}$ & $26.28^{\mathrm{a}}$ & 1.08 & 0.0052 \\
Plasma IgM, mg/mL & $11.1^{\mathrm{b}}$ & $17.86^{\mathrm{a}}$ & 1.12 & 0.0004 \\
\hline
\end{tabular}


Table 1. Cont.

\begin{tabular}{ccccc}
\hline Item & Control & Treatment & SEM & $p$-Value \\
\hline Day 35 & & & & \\
Fecal sIgA, $\mu \mathrm{g} / \mathrm{g}$ & $132.09^{\mathrm{b}}$ & $148.78^{\mathrm{a}}$ & 3.25 & 0.0071 \\
Plasma IgG, mg/mL & $13.74^{\mathrm{b}}$ & $24.08^{\mathrm{a}}$ & 1.22 & $<0.0001$ \\
Plasma IgM, mg/mL & $10.95^{\mathrm{b}}$ & $18.03^{\mathrm{a}}$ & 0.95 & $<0.0001$ \\
Day 56 & & & & \\
Fecal sIgA, $\mu \mathrm{g} / \mathrm{g}$ & $102.24^{\mathrm{b}}$ & $128.12^{\mathrm{a}}$ & 3.85 & 0.0001 \\
Plasma IgG, mg/mL & $16.92^{\mathrm{b}}$ & $19.05^{\mathrm{a}}$ & 0.41 & 0.0058 \\
Plasma IgM, mg/mL & $9.91^{\mathrm{b}}$ & $13.69^{\mathrm{a}}$ & 0.58 & $<0.0001$
\end{tabular}

$\mathrm{a}, \mathrm{b} \overline{\text { Values within a row with different superscripts differ significantly at } p<0.05 \text {, All values are presented as means }}$ \pm SEM.

\subsection{Early-Life Intervention with FMT-CS Affected the Efficacy of Vaccines}

To assess the impact of early-life intervention with FMT-CS on vaccine responses in piglets, we investigated the antibody responses to four vaccines that are frequently applied to piglets. As shown in Figure 6, the antibodies of M.hyo (Figure 8A) and PCV2 (Figure 8B) were increased in treated piglets, while no differences were detected for anti-CSFV antibody (Figure 8C) and anti-PRV antibody (Figure 8D).

A
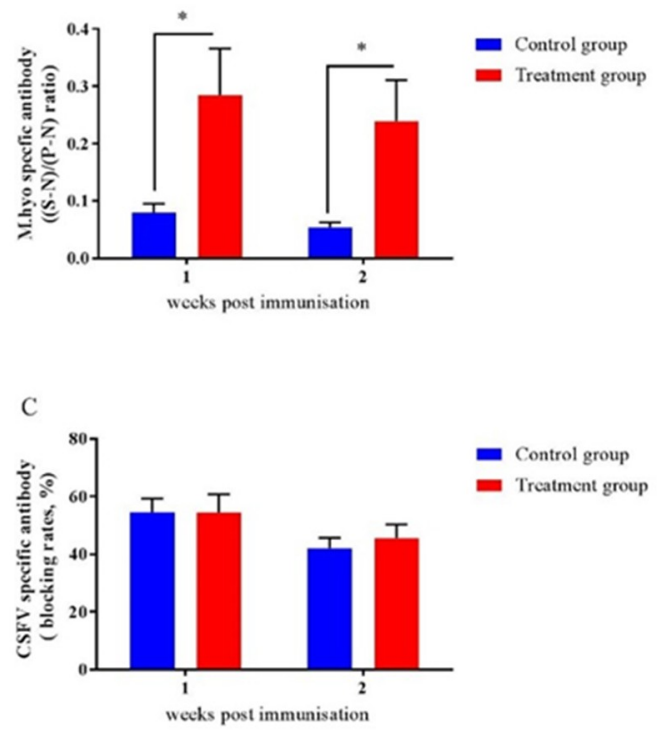

$\mathrm{B}$
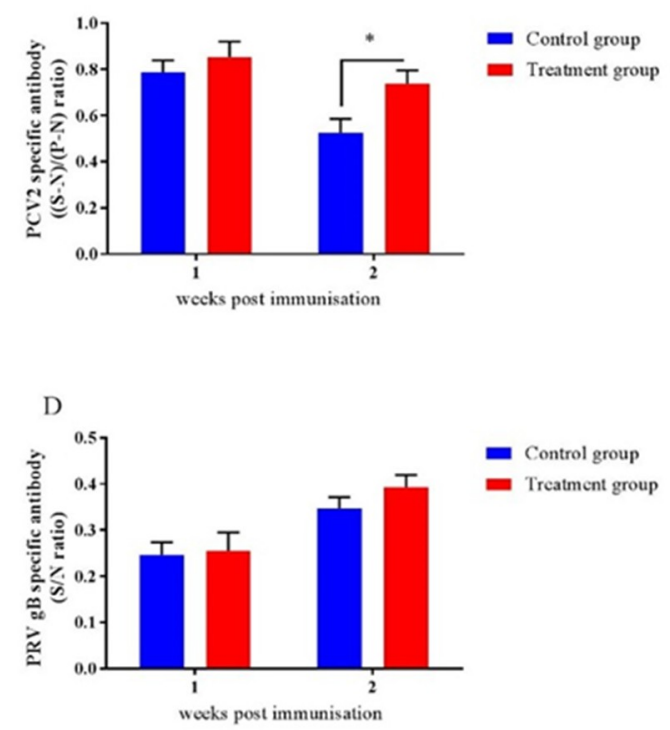

Figure 8. Effects of early-life intervention with FMT-CS on vaccine antibody responses. Commercial ELISA kits were utilized to test anti-M.hyo antibody (A), anti-PCV2 antibody (B), anti-CSFV antibody (C), and anti-PRV antibody (D). (S-N)/(P-N) ratio: S, sample OD630; N, negative control OD630;

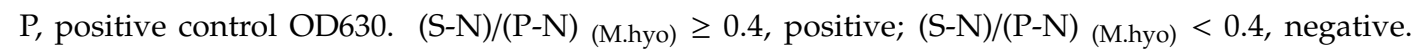
$(\mathrm{S}-\mathrm{N}) /(\mathrm{P}-\mathrm{N})_{(\mathrm{PCV} 2)} \geq 0.4$, positive; $(\mathrm{S}-\mathrm{N}) /(\mathrm{P}-\mathrm{N})_{(\mathrm{PCV} 2)}<0.4$, negative. Blocking rate $=$ (negative control OD450-sample OD450)/negative control OD450. Blocking rate $(\mathrm{CSFV}) \geq 40 \%$, positive; blocking rate $(\mathrm{CSFV})<40 \%$, negative. $\mathrm{S} / \mathrm{N}$ ratio = sample OD450/negative control OD450. S/N ${ }_{(\mathrm{PRV} \mathrm{gB})} \leq 0.6$, positive; $\mathrm{S} / \mathrm{N}_{(\mathrm{PRV} g B)}>0.6$, negative. Values are expressed as mean $\pm \mathrm{SEM} .{ }^{*} p<0.05,{ }^{* *} p<0.01,{ }^{* * *} p<0.001$.

\subsection{Early-Life Intervention with FMT-CS Affected Weaning Stress}

In order to evaluate the effect of FMT-CS on weaning stress, the antioxidant status, intestinal barrier function, and inflammation levels were determined in piglets after weaning (at the age of $35 \mathrm{~d}$ ). Table 2 shows the effects of early intervention with FMT-CS on CAT, GSH-px, MDA, T-AOC, and T-SOD in the plasma of piglets at the age of $35 \mathrm{~d}$. There were significant increases in CAT $(p<0.05)$, 
GSH-px $(p=0.0514)$, T-AOC $(p<0.05)$, and T-SOD $(p<0.05)$ levels, and a significant decrease in plasma MDA $(p<0.05)$, suggesting that early intervention with FMT-CS can enhance the antioxidant capacity of piglets after weaning. Besides, FMT-CS significantly reduced the levels of DAO and D-LA in the plasma, indicating that early intervention with FMT-CS may improve the intestinal barrier function of the piglets after weaning. The treatment significantly decreased the levels of TNF- $\alpha$ and IL- 6 in the plasma, and enhanced the level of anti-inflammatory factor IL-10. Moreover, a significant decline in plasma cortisol level was detected in the treatment group, indicating that early intervention with FMT-CS may suppress the inflammation in piglets after weaning.

Table 2. Effect of early-life intervention with FMT-CS on weaning stress of piglets.

\begin{tabular}{|c|c|c|c|c|c|}
\hline Items $^{1}$ & Biomarkers & Control & Treatment & SEM & $p$-Value \\
\hline \multirow{5}{*}{ Antioxidant Indices } & CAT, U/mL & $25.14^{b}$ & $33.22^{a}$ & 1.81 & 0.0218 \\
\hline & GSH-px, U/mL & 368.38 & 469.15 & 26.12 & 0.0514 \\
\hline & $\mathrm{MDA}, \mathrm{nmol} / \mathrm{mL}$ & $7.81^{\mathrm{a}}$ & $5.30^{\mathrm{b}}$ & 0.53 & 0.0147 \\
\hline & T-AOC, U/mL & $36.22^{b}$ & $48.70^{\mathrm{a}}$ & 2.94 & 0.0302 \\
\hline & T-SOD, U/mL & $14.58^{\mathrm{b}}$ & $35.59^{\mathrm{a}}$ & 1.44 & 0.0271 \\
\hline \multirow{2}{*}{ Intestinal Barrier Biomarkers } & $\mathrm{DAO}, \mathrm{ng} / \mathrm{mL}$ & $374.18^{a}$ & $296.8^{\mathrm{b}}$ & 17.513 & 0.0115 \\
\hline & D-LA, umol/mL & $880.16^{\mathrm{a}}$ & $631.27^{\mathrm{b}}$ & 19.84 & 0.0004 \\
\hline \multirow{6}{*}{ Inflammation Levels } & IFN- $\gamma, \mathrm{pg} / \mathrm{mL}$ & 70.99 & 59.43 & 2.72 & 0.0530 \\
\hline & $\mathrm{TNF}-\alpha, \mathrm{pg} / \mathrm{mL}$ & $443.13^{\mathrm{a}}$ & $245.89^{b}$ & 9.42 & $<0.0001$ \\
\hline & IL-6, pg/mL & $1152.04^{\mathrm{a}}$ & $900.18^{b}$ & 34.09 & 0.0044 \\
\hline & IL-2, pg/mL & 610.79 & 729.64 & 36.86 & 0.1640 \\
\hline & IL-10, pg/mL & 374.69 & 493.06 & 27.96 & 0.0713 \\
\hline & Cortisol, ng/mL & $272.73^{\mathrm{a}}$ & $210.05^{b}$ & 8.59 & 0.0022 \\
\hline
\end{tabular}

${ }^{1}$ All of these biomarkers were determined at the age of $35 \mathrm{~d}$. ${ }^{\mathrm{a}, \mathrm{b}}$ Values within a row with different superscripts differ significantly at $p<0.05$, All values are presented as means \pm SEM.

\section{Discussion}

Recently, a number of studies have demonstrated an association between the immune system and gut microbiota [20,32-37]. Early infancy is considered as an ideal window for gut microbial colonization [18,38]. Our previous study has validated the benefits of transplanting sow fecal microbiota to newborn piglets [20]. Here, by combining the oral administration of fecal microbiota of gestation sows with C. butyricum and S. boulardii in newborn piglets, this study for the first time focuses on the promotion of immune development through improving the gut microbiota of piglets. Our results indicated that early intervention with FMT-CS did promote the host immune maturation, and the development of the host immune system was consistent with that of intestinal microbiota.

\subsection{Early-Life Intervention with FMT-CS Promotes the Maturation of Gut Microbiota}

The results showed that early-life intervention with FMT-CS significantly increased the alpha diversity of gut microbiota during the suckling period, representing the positive effect of FMT-CS on the development of gut microbiota during the early life period of piglets [39]. Previous studies have shown that FMT or dietary C. butyricum can promote the maturation of intestinal bacteria, which is in accordance with our results $[35,40]$. In addition, PICRUSt analysis showed that the genes related to the metabolism of vitamins, energy, and amino acids, which represent bacterial maturity [30,31,41], were up-regulated in treated piglets at the age of $7 \mathrm{~d}$. From our results, it can be inferred that early intervention with FMT-CS may accelerate gut microbiota maturation in newborn piglets.

\subsection{Early-life Intervention with FMT-CS Promotes the Development and Function of Immune System}

It has been confirmed that gut microbiota plays a critical role in the development of immune system $[18,34]$, especially the innate immune system [11,42]. We determined several indicators, such as IL-22, IL-17, and IFN- $\gamma$, to examine the development of the innate immune system $[43,44]$. The 
significant increase in plasma IL-22 and IL-17 may indicate the development of the innate immune system $[45,46]$. Previous studies have confirmed that the maturation of the intestinal innate immune system of mammals occurs in infancy [7-9]. Here, the evaluation of the developmental changes in innate immunity based on the plasma levels of cytokines at different ages revealed that the early intervention with FMT-CS can promote the innate immunity of piglets during the period from birth to weaning.

On the other hand, innate immunity plays an important role in connecting gut microbiota with adaptive immune system development $[33,47]$. In this study, biomarkers of adaptive immune system were significantly increased in treated piglets. SIgA is produced by gut plasma cells [33]. Gastrointestinal sIgA plays a crucial role in maintaining the intestinal epithelial barrier [48]. It has been shown that fecal sIgA can be quantified as a biomarker of intestinal permeability and immune functions [48]. Besides, early intervention with FMT-CS increased plasma IgG and IgM, and similar results were reported in previous studies of suckling piglets $[20,35]$. Our results indicate that early intervention with FMT-CS can improve the adaptive immune function of piglets.

Furthermore, it has been indicated that early-life gut microbiota dysbiosis damages vaccine immune responses [49,50]. In this study, we found that early-life intervention with FMT-CS can reduce the risk of gut microbiota dysbiosis, as well as improve the antibody levels of M.hyo and PCV2. The M.hyo and PCV2 vaccines are inoculated during the suckling period of piglets, while the CSFV and PRV vaccines are applied during the post-weaning period, indicating that gut microbiota intervention improves vaccine efficacy mainly in the early-life period. It is worth noting that a significant increase was detected in plasma IgG concentration at the age of $56 \mathrm{~d}$ after early intervention. However, there was no significant increase in vaccine efficacy after weaning, which may be due to the fact that IgG and IgM contents are correlated with mucosal humoral immunity [31,51], while specific antibodies are associated with the animals' tolerance to specific antigens.

In summary, the early intervention of FMT-CS can promote the innate immune development, enhance the acquired immune function, and improve the vaccine efficacy during the suckling period.

\subsection{Early Intervention with FMT-CS Increases Beneficial Bacteria and Decreases the Opportunistic Pathogens of Piglets}

Firmicutes and Bacteroidetes are the core pig microbiome [39,52]. BugBase analysis revealed a slight decrease in aerobes at the age of $7 \mathrm{~d}$, indicating a faster oxygen consumption in the intestines after FMT-CS treatment, which may contribute to the colonization of commensal microflora [21,22]. Similar to the results in previous studies, early intervention with FMT-CS significantly increased the beneficial bacteria and decreased the harmful bacteria [40,53-56]. The relative abundance of the producers of short-chain fatty acids, such as Oscillospira, Phascolarctobacterium, Blautia, Coprococcus and Faecalibacterium, was significantly increased, possibly promoting the immune and anti-stress functions $[57,58]$. Therefore, the piglets may have better ability to cope with the damage caused by weaning stress. Lactobacillus plays a key role in disease prevention [59]. After weaning, Lactobacillus is the commensal bacteria and is widely used as a probiotic. Fecal Lactobacillus is associated with the regulation of the gut immune function and maintenance of the balance of gut microbiota to reduce the inflammatory responses [56,60-63]. Recent studies have confirmed that FMT could enrich the abundance of gut Lactobacillus, which may contribute to the improvement of the intestinal barrier and reduction of diarrhea incidence in piglets $[20,35,53]$. Moreover, Lactobacillus was reported to enhance the gut barrier to confer diarrhea resistance after the weaning of piglets [64]. Therefore, after weaning, significant declines were detected in plasma DAO and D-LA, which are regarded as indicators of intestinal barrier function, suggesting that the piglets in the treatment group developed a stronger intestinal barrier function [65-67]. Moreover, the treated piglets showed significant increases in Lachnospira, Bacillus, and Lysinibacillus, which were found to be associated with the maintenance of gut health and promotion of growth $[63,68]$. In addition, BugBase analysis showed significant decreases of pathogens in treated piglets, which is consistent with the results of the lefSe and metastats analyses. 
Potential pathogens, such as Fusobacteriaceae, Lachnospiraceae, Enterobacteriaceae, and Akkermansia, were significantly decreased in treated piglets, indicating a lower occurrence rate of intestinal dysbiosis [50]. The above results suggest that early-life intervention with FMT-CS increases the relative abundance of beneficial bacteria, while decreases that of opportunistic pathogens.

\subsection{Early-Life Intervention with FMT-CS Confers Weaning Stress Resistance to Piglets}

Previous studies have suggested that weaning can cause oxidative stress-induced damage and inflammation in piglets [69-71], which can negatively affect the growth performance of weaned pigs [72]. The gut microbiota plays a vital role in regulating the weaning stress of piglets [64,73]. In order to evaluate to which extent weaning stress may affect the physiological and immune status of piglets, oxidative stress indicators and inflammatory factors were measured. Recent studies have proposed that FMT may be an effective therapeutic approach to eliminate oxidative stress [74]. In this regard, our results also suggest that early intervention with FMT may have a fairly lasting effect on oxidative stress caused by weaning. Moreover, it has been found that FMT can reverse the inflammatory state caused by depression [75]. Besides, early intervention with FMT-CS also tended to increase the plasma level of IL-2, an important survival and proliferation-inducing cytokine for Treg cells [76,77]. In this study, the results demonstrated that early intervention with FMT-CS can suppress the inflammation level in piglets caused by weaning. Overall, it can be concluded that weaning stress can be alleviated by the early intervention with FMT-CS in piglets.

\section{Materials and Methods}

\subsection{Preparation of Fecal Microbiota Suspension}

Six healthy gestation sows (on day 70 of gestation, Landrace $\times$ Large white), which had no recent history of gastrointestinal diseases and antibiotics treatment in more than 2 months, were used as fecal donors in this experiment. The fresh fecal of donor sows was collected separately after $12 \mathrm{~h}$ of fasting. The fecal suspension was prepared as described by Pang et al. (2007) [78]. In brief, fresh fecal sample was immediately diluted 20 -fold in $0.1 \mathrm{M}$ sterile phosphate-buffered saline containing $10 \%$ glycerol (PBSG). Then, $1.0 \times 10^{9} \mathrm{CFU} / \mathrm{mL}$ C. butyricum and $1.0 \times 10^{9} \mathrm{CFU} / \mathrm{mL}$ S. boulardii were added into the fecal suspension, which was used as the transplanted bacterial suspension (TBS) and stored at $-80^{\circ} \mathrm{C}$.

\subsection{Animal Experimental Design}

The protocol for the animal experimental procedures was approved by Institutional Animal Care and Use Committee of Huazhong Agricultural University (Wuhan, China). The ethical number of this study is HZAUSW-2018-013.

One hundred and ninety healthy neonatal piglets (Duroc $\times$ Landrace $\times$ Large white) with an average birth weight of $1.55 \pm 0.31 \mathrm{~kg}$ were obtained from 22 sows with the parity between 2 and 5 . All piglets were raised under the same conditions on a commercial farm in Guangxi Province, China. In order to avoid maternal differences, the piglets from 22 litters were randomly divided into two groups: the control group $\left(n_{\text {sows }}=10, n_{\text {piglets }}=84\right)$ and the treatment group $\left(n_{\text {sows }}=12, n_{\text {piglets }}=106\right)$. In the first three days after birth, each piglet in the treatment group was orally administered with TBS daily at the volume of $2 \mathrm{~mL}$, while the piglets in the control group were given the same frequency and volume of PBSG. Piglets were weaned at the age of $28 \mathrm{~d}$. A total of 149 weaned piglets $\left(n_{\text {control }}=71, n_{\text {treatment }}=78\right)$ were randomly raised in eight similar pens, with four pens for each group. The experiment was ended at the age of $56 \mathrm{~d}$. During the experiment period, the piglets were allowed free access to water and antibiotics-free diets.

\subsection{Immunization}

The piglets were immunized with the following inactivated vaccines: mycoplasma hyopneumoniae (M.hyo) vaccine at the age of $8 \mathrm{~d}$, porcine circovirus type 2 (PCV2) vaccine at the age of $15 \mathrm{~d}$, classical 
swine fever virus (CSFV) vaccine at the age of $42 \mathrm{~d}$, and swine pseudorabies virus (PRV) vaccine at the age of $49 \mathrm{~d}$.

\subsection{Sample Collection}

Blood samples were collected from 12 randomly selected piglets from the control and treatment groups by the vena jugularis into heparin vacuum blood tubes. The samples were then centrifuged at $3000 \times g$ for $30 \mathrm{~min}$, and the plasma was carefully separated and stored at $-80^{\circ} \mathrm{C}$ until analysis. Fecal samples were collected at the age of $7 \mathrm{~d}$ and $56 \mathrm{~d}$ from 12 randomly selected piglets of the control and treatment groups, and stored at $-80{ }^{\circ} \mathrm{C}$ until analysis.

\subsection{DNA Extraction, 16S rRNA Gene Amplification, and Illumina MiSeq Sequencing}

Microbial DNA was extracted from intestinal contents using a QIAamp DNA Stool Mini Kit (Qiagen, Germany) following the manufacturer's protocols. Successful DNA extraction was confirmed by $0.8 \%$ agarose-gel electrophoresis. The V3-V4 hypervariable region of the bacterial $16 \mathrm{~S}$ rRNA gene was amplified using primers 341F ( $5^{\prime}$-ACT CCT ACG GGA GGC AGC AG-3') and 806R (5'-GGA CTA CHV GGG TWT CTA AT- $3^{\prime}$ ). The PCR conditions were predenaturation at $98^{\circ} \mathrm{C}$ for $2 \mathrm{~min}, 25$ cycles of denaturation at $98{ }^{\circ} \mathrm{C}$ for $15 \mathrm{~s}$, annealing at $55^{\circ} \mathrm{C}$ for $30 \mathrm{~s}$, elongation at $72{ }^{\circ} \mathrm{C}$ for $30 \mathrm{~s}$, and a final post-elongation cycle at $72{ }^{\circ} \mathrm{C}$ for $5 \mathrm{~min}$. The PCR products were purified with AMPure XP beads (AXYGEN). After purification, the PCR products were used for the construction of libraries and then paired-end sequenced on Illumina Miseq (Illumina, CA, USA) at the Personalbio, Shanghai, China.

\subsection{Analysis of Sequencing Data}

Alpha diversity (including four indices: Chao1, Shannon, Simpson, and ACE) of bacterial richness and diversity was analyzed using QIIME (Caporaso et al., 2010). Beta diversity was analyzed by PCA based on operational taxonomic unit (OTU), and the hierarchical clustering tree was constructed based on unweighted Unifrac distances. Bray-Curtis distances were calculated based on the square root-transformed OTU relative abundance and used to generate the non-metric multidimensional scaling (NMDS) ordination plot. All of these indices in our samples were calculated with QIIME (Version 1.7.0) and displayed with R software (Version 2.15.3). On the basis of classified information, a histogram of linear discriminant analysis (LDA) distribution was implemented by the LDA effect size analysis (LEfSe) to find the bacteria with significant differences in relative abundance between two groups. Microbial phenotypes were predicted with BugBase [79], a software that relies on the tools PICRUSt, IMG, KEGG and PATRIC.

\subsection{Measurements of Cytokines Related to Innate and Adaptive Immunity Development and Antibody Responses}

sIgA, IgM, IgG, IL-22, IL-17, and IFN- $\gamma$ were measured by ELISA kits (mlbio good elisakit producers, Shanghai, China) following the manufacture's protocol. Antigen-specific IgG was measured using commercially available ELISA kits including the M.hyo IgG ELISA kit (IDEXX Laboratories, GP521, America), PCV2 IgG ELISA kit (zcwdbio, 180801, Wuhan, China), CSFV IgG ELISA kit (IDEXX Laboratories, M301, America), and PRV IgG ELISA kit (Keqian Biology Co., Ltd., 181010, Wuhan, China) following the manufacture's protocol. Data were analyzed using GraphPad Prism 7.01 (GraphPad Software Inc., La Jolla, CA, USA). A two-tailed Student's $t$ test was used to assess the significance.

\subsection{Intestinal Barrier Function}

The levels of plasma diamine oxidase (DAO) and D-lactate (D-LA) were examined using an ELISA test kit (m1002413 and m1647900, mlbio good elisakit producers, Shanghai, China) according to the manufacturer's instructions. The absorbance was determined with a 96-well microtiter plate reader INFINITE 200 PRO (Tecan Austrla Gmbh, Untersbergstr. 1A, A-2082 Grödlg, Austria). 


\subsection{Antioxidant Indices}

Glutathione peroxidase (GSH-px), catalase (CAT), total antioxidant capacity (T-AOC), malondialdehyde (MDA), and total superoxide dismutase (T-SOD) of plasma were examined using an assay kit (Nanjing Jiancheng Bioengineering Institute (Nanjing, China)) according to the manufacturer's instructions.

\subsection{Inflammation Levels}

The contents of plasma cortisol, pro-inflammatory cytokines (Plasma INF- $\gamma$, TNF- $\alpha$, and IL-6), and anti-inflammatory cytokines (plasma IL-2 and IL-10) were examined using an ELISA test kit (mlbio good elisakit producers, Shanghai, China) according to the manufacturer's instructions.

\subsection{Statistical Analysis}

Before the analysis, Kolmogorov-Smirnov and Levene tests were performed for the normality and heteroscedasticity of the data. Cytokine values were assessed by ANOVA. The procedure for the repeated measurements of SAS 9.4 (SAS Institute, Inc., Cary, NC, US) was used. Data were expressed as means \pm SEM. Statistical significance was defined as $p<0.05$, whereas $p$ values between 0.05 and 0.10 were considered as a trend.

\section{Conclusions}

Our results reveal that the early-life intervention with FMT-CS regulates gut microbiota structure, accelerates the maturation of gut microbiota, and reduces the risk of gut microbiota dysbiosis in piglets. We also found that FMT-CS can promote the development of the immune system and increase vaccine efficacy during the early-life period of piglets, so as to alleviate the damage of weaning stress. Our findings provide an important reference for the regulation of innate and adaptive immunity development, vaccine efficacy, and weaning stress through early-life gut microbiota intervention in the pig industry. However, further studies are still needed to better understand the mechanism by which gut microbiota regulates the development of innate immune cells.

Supplementary Materials: Supplementary materials can be found at http://www.mdpi.com/1422-0067/21/2/503/s1.

Author Contributions: The author' contributions are as follows: J.P. was in charge of the whole trial; Q.X. analyzed experimental data and wrote the manuscript; Q.X., X.W., Y.P., L.W., Y.G. and C.C. for animal feeding and sample collections; Q.X. and L.Z. assisted with laboratory analyses; All authors read and approved the final manuscript.

Funding: This study was funded by the National Natural Science Foundation of China (31772609); Hubei Agricultural Sciences and Technology Innovation Center (2019-620-000-001-30); China Agriculture Research System (CARS-36).

Acknowledgments: We thank Huazhong Agricultural University, Guangxi Yangxiang Co., Ltd. (China), and Shanghai Personal Biotechnology Co., Ltd. (China) for technical assistance.

Conflicts of Interest: There is no conflict of interest.

\section{Abbreviations}

$\begin{array}{ll}\text { FMT } & \text { Fecal microbiota transplantation } \\ \text { FMT-CS } & \text { FMT combined with C. butyricum and S. boulardii } \\ \text { GSH-px } & \text { Glutathione peroxidase } \\ \text { CAT } & \text { Catalase } \\ \text { T-AOC } & \text { Total antioxidant capacity } \\ \text { MDA } & \text { Malondialdehyde } \\ \text { T-SOD } & \text { Total superoxide dismutase } \\ \text { D-LA } & \text { D-lactate } \\ \text { DAO } & \text { Diamine oxidase } \\ \text { PBSG } & \text { Phosphate-buffered saline containing 10\% glycerol } \\ \text { TBS } & \text { Transplanted bacterial suspension }\end{array}$




\section{References}

1. Brandtzaeg, P. Mucosal immunity: Integration between mother and the breast-fed infant. Vaccine 2003, 21, 3382-3388. [CrossRef]

2. Georgountzou, A.; Papadopoulos, N.G. Postnatal innate immune development: From birth to adulthood. Front. Immunol. 2017, 8, 957. [CrossRef] [PubMed]

3. Yang, Q.; Huang, X.; Wang, P.; Yan, Z.; Sun, W.; Zhao, S.; Gun, S. Longitudinal development of the gut microbiota in healthy and diarrheic piglets induced by age-related dietary changes. MicrobiologyOpen 2019. [CrossRef] [PubMed]

4. Zinkernagel, R.M. Maternal antibodies, childhood infections, and autoimmune diseases. N. Engl. J. Med. 2001, 345, 1331. [CrossRef] [PubMed]

5. Chen, L.; Li, S.; Zheng, J.; Li, W.; Jiang, X.; Zhao, X.; Li, J.; Che, L.; Lin, Y.; Xu, S. Effects of dietary clostridium butyricum supplementation on growth performance, intestinal development, and immune response of weaned piglets challenged with lipopolysaccharide. J. Anim. Sci. Biotechnol. 2018, 9, 211-224. [CrossRef] [PubMed]

6. Stras, S.F.; Werner, L.; Toothaker, J.M.; Olaloye, O.O.; Oldham, A.L.; McCourt, C.C.; Lee, Y.N.; Rechavi, E.; Shouval, D.S.; Konnikova, L. Maturation of the human intestinal immune system occurs early in fetal development. Dev. Cell 2019, 51, 357-373. [CrossRef]

7. Renz, H.; Brandtzaeg, P.; Hornef, M. The impact of perinatal immune development on mucosal homeostasis and chronic inflammation. Nat. Rev. Immunol. 2011, 12, 9-23. [CrossRef]

8. Jain, N.; Walker, W.A. Diet and host-microbial crosstalk in postnatal intestinal immune homeostasis. Nat. Rev. Gastroenterol. Hepatol. 2015, 12, 14-25. [CrossRef]

9. De Gomez, A.M.; Ganal-Vonarburg, S.C.; Fuhrer, T.; Rupp, S.; Uchimura, Y.; Li, H.; Steinert, A.; Heikenwalder, M.; Hapfelmeier, S.; Sauer, U. The maternal microbiota drives early postnatal innate immune development. Science 2016, 351, 1296. [CrossRef]

10. Hachung, C.; Pamp, S.J.; Hill, J.A.; Surana, N.K.; Edelman, S.M.; Troy, E.B.; Reading, N.C.; Villablanca, E.J.; Sen, W.; Mora, J.R. Gut immune maturation depends on colonization with a host-specific microbiota. Cell 2012, 149, 1578-1593.

11. Sanos, S.; Bui, V.A.; Oberle, K.; Heners, C.; Johner, C.; Diefenbach, A. Rorgammat and commensal microflora are required for the differentiation of mucosal interleukin 22-producing nkp46+ cells. Nat. Immunol. 2009, 10, 83-91. [CrossRef] [PubMed]

12. Li, S.S.; Zhu, A.; Benes, V.; Costea, P.I.; Hercog, R.; Hildebrand, F.; Huerta-Cepas, J.; Nieuwdorp, M.; Salojarvi, J.; Voigt, A.Y.; et al. Durable coexistence of donor and recipient strains after fecal microbiota transplantation. Science 2016, 352, 586-589. [CrossRef] [PubMed]

13. Litvak, Y.; Baumler, A.J. The founder hypothesis: A basis for microbiota resistance, diversity in taxa carriage, and colonization resistance against pathogens. PLoS Pathog. 2019, 15, e1007563. [CrossRef] [PubMed]

14. Martinez, I.; Maldonado-Gomez, M.X.; Gomes-Neto, J.C.; Kittana, H.; Ding, H.; Schmaltz, R.; Joglekar, P.; Cardona, R.J.; Marsteller, N.L.; Kembel, S.W.; et al. Experimental evaluation of the importance of colonization history in early-life gut microbiota assembly. Elife 2018, 7, e36521. [CrossRef]

15. Al Nabhani, Z.; Eberl, G. GAPs in early life facilitate immune tolerance. Sci. Immunol. 2017, 2. [CrossRef]

16. El, A.S.; Derrien, M.; Merrifield, C.A.; Levenez, F.; Doré, J.; Boekschoten, M.V.; Dekker, J.; Holmes, E.; Zoetendal, E.G.; Van, B.P. Gut bacteria-host metabolic interplay during conventionalisation of the mouse germfree colon. ISME J. 2013, 7, 743-755.

17. El, A.S.; Hooiveld, G.; Tremaroli, V.; Bäckhed, F.; Kleerebezem, M. The gut microbiota and mucosal homeostasis: Colonized at birth or at adulthood, does it matter? Gut Microbes 2013, 4, 118-124.

18. Gensollen, T.; Iyer, S.S.; Kasper, D.L.; Blumberg, R.S. How colonization by microbiota in early life shapes the immune system. Science 2016, 352, 539-544. [CrossRef]

19. Rey-Santano, C.; Mielgo, V.; Valls-i-Soler, A.; Encinas, E.; Lukas, J.C.; Vozmediano, V.; Suarez, E. Evaluation of fentanyl disposition and effects in newborn piglets as an experimental model for human neonates. PLoS ONE 2014, 9, e90728. [CrossRef]

20. Cheng, C.S.; Wei, H.K.; Wang, P.; Yu, H.C.; Zhang, X.M.; Jiang, S.W.; Peng, J. Early intervention with faecal microbiota transplantation: An effective means to improve growth performance and the intestinal development of suckling piglets. Anim. Int. J. Anim. Biosci. 2019, 13, 533-541. [CrossRef] 
21. Espey, M.G. Role of oxygen gradients in shaping redox relationships between the human intestine and its microbiota. Free Radic. Biol. Med. 2013, 55, 130-140. [CrossRef] [PubMed]

22. Kim, Y.G.; Sakamoto, K.; Seo, S.U.; Pickard, J.M.; Gillilland, M.G., 3rd; Pudlo, N.A.; Hoostal, M.; Li, X.; Wang, T.D.; Feehley, T.; et al. Neonatal acquisition of clostridia species protects against colonization by bacterial pathogens. Science 2017, 356, 315-319. [CrossRef] [PubMed]

23. Hudson, L.E.; McDermott, C.D.; Stewart, T.P.; Hudson, W.H.; Rios, D.; Fasken, M.B.; Corbett, A.H.; Lamb, T.J. Characterization of the probiotic yeast saccharomyces boulardii in the healthy mucosal immune system. PLOS ONE 2016, 11, e0153351. [CrossRef]

24. Hancox, L.R.; Le Bon, M.; Richards, P.J.; Guillou, D.; Dodd, C.E.; Mellits, K.H. Effect of a single dose of Saccharomyces cerevisiae var. boulardii on the occurrence of porcine neonatal diarrhoea. Anim. Int. J. Anim. Biosci. 2015, 9, 1756-1759. [CrossRef] [PubMed]

25. Stier, H.; Bischoff, S.C. Saccharomyces boulardii cncm i-745 influences the gut-associated immune system. MMW Fortschr. Med. 2017, 159, 1-6. [CrossRef] [PubMed]

26. Zhang, S.F.; Tang, Z.S.; Ling, T.; Tao, X.X.; Suo, Q.F.; Xu, X.M. Effects of clostridium butyricum and bifidobacterium on btla expression on $\mathrm{cd} 4+\mathrm{t}$ cells and lymphocyte differentiation in late preterm infants. Microb. Pathog. 2016, 100, 112-118. [CrossRef]

27. Ling, Z.; Liu, X.; Cheng, Y.; Luo, Y.; Yuan, L.; Li, L.; Xiang, C. Clostridium butyricum combined with bifidobacterium infantis probiotic mixture restores fecal microbiota and attenuates systemic inflammation in mice with antibiotic-associated diarrhea. Biomed. Res. Int. 2015, 2015, 582048. [CrossRef]

28. Wang, K.; Cao, G.; Zhang, H.; Li, Q.; Yang, C. Effects of Clostridium butyricum and Enterococcus faecalis on growth performance, immune function, intestinal morphology, volatile fatty acids, and intestinal flora in a piglet model. Food Funct 2019, 10, 7844-7854. [CrossRef]

29. Langille, M.G.I.; Zaneveld, J.; Caporaso, J.G.; McDonald, D.; Knights, D.; Reyes, J.A.; Clemente, J.C.; Burkepile, D.E.; Thurber, R.L.V.; Knight, R.; et al. Predictive functional profiling of microbial communities using 16s rrna marker gene sequences. Nat. Biotechnol. 2013, 31, 814. [CrossRef]

30. Hu, J.; Nie, Y.; Chen, J.; Zhang, Y.; Wang, Z.; Fan, Q.; Yan, X. Gradual changes of gut microbiota in weaned miniature piglets. Front. Microbiol. 2016, 7, 1727. [CrossRef]

31. Wang, T.; Teng, K.; Liu, Y.; Shi, W.; Zhang, J.; Dong, E.; Zhang, X.; Tao, Y.; Zhong, J. Lactobacillus plantarum pfm 105 promotes intestinal development through modulation of gut microbiota in weaning piglets. Front. Microbiol. 2019, 10, 90. [CrossRef] [PubMed]

32. Geng, S.; Cheng, S.; Li, Y.; Wen, Z.; Ma, X.; Jiang, X.; Wang, Y.; Han, X. Faecal microbiota transplantation reduces susceptibility to epithelial injury and modulates tryptophan metabolism of the microbial community in a piglet model. J. Crohns Colitis 2018, 12, 1359-1374. [CrossRef] [PubMed]

33. Honda, K.; Littman, D.R. The microbiota in adaptive immune homeostasis and disease. Nature 2016, 535, 75-84. [CrossRef] [PubMed]

34. Hooper, L.V.; Littman, D.R.; Macpherson, A.J. Interactions between the microbiota and the immune system. Science 2012, 336, 1268-1273. [CrossRef]

35. Hu, L.; Geng, S.; Li, Y.; Cheng, S.; Fu, X.; Yue, X.; Han, X. Exogenous fecal microbiota transplantation from local adult pigs to crossbred newborn piglets. Front. Microbiol. 2017, 8, 2663. [CrossRef]

36. Ouwehand, A.C.; Bergsma, N.; Parhiala, R.; Lahtinen, S.; Gueimonde, M.; Finne-Soveri, H.; Strandberg, T.; Pitkala, K.; Salminen, S. Bifidobacterium microbiota and parameters of immune function in elderly subjects. FEMS Immunol. Med. Microbiol. 2008, 53, 18-25. [CrossRef]

37. Thaiss, C.A.; Zmora, N.; Levy, M.; Elinav, E. The microbiome and innate immunity. Nature 2016, 535, 65-74. [CrossRef]

38. Nguyen, Q.N.; Himes, J.E.; Martinez, D.R.; Permar, S.R. The impact of the gut microbiota on humoral immunity to pathogens and vaccination in early infancy. PLoS Pathog. 2016, 12, e1005997. [CrossRef]

39. Slifierz, M.J.; Friendship, R.M.; Weese, J.S. Longitudinal study of the early-life fecal and nasal microbiotas of the domestic pig. BMC Microbiol. 2015, 15, 184. [CrossRef]

40. Zhang, J.; Chen, X.; Liu, P.; Zhao, J.; Sun, J.; Guan, W.; Johnston, L.J.; Levesque, C.L.; Fan, P.; He, T.; et al. Dietary clostridium butyricum induces a phased shift in fecal microbiota structure and increases the acetic acid-producing bacteria in a weaned piglet model. J. Agric. Food Chem. 2018, 66, 5157-5166. [CrossRef]

41. McDonald, E. Vitamins and cofactors: Highlights of esboc 2009. Nat. Chem. Biol. 2009, 5, 530-533. [CrossRef] [PubMed] 
42. Sawa, S.; Cherrier, M.; Lochner, M.; Satoh-Takayama, N.; Fehling, H.J.; Langa, F.; Di Santo, J.P.; Eberl, G. Lineage relationship analysis of rorgammat+ innate lymphoid cells. Science 2010, 330, 665-669. [CrossRef] [PubMed]

43. Bachmann, M.; Horn, K.; Rudloff, I.; Goren, I.; Holdener, M.; Christen, U.; Darsow, N.; Hunfeld, K.P.; Koehl, U.; Kind, P.; et al. Early production of il-22 but not il-17 by peripheral blood mononuclear cells exposed to live borrelia burgdorferi: The role of monocytes and interleukin-1. PLoS Pathog. 2010, 6, e1001144. [CrossRef] [PubMed]

44. Vivier, E.; Artis, D.; Colonna, M.; Diefenbach, A.; Di Santo, J.P.; Eberl, G.; Koyasu, S.; Locksley, R.M.; McKenzie, A.N.J.; Mebius, R.E.; et al. Innate lymphoid cells: 10 years on. Cell 2018, 174, 1054-1066. [CrossRef]

45. Atarashi, K.; Tanoue, T.; Ando, M.; Kamada, N.; Nagano, Y.; Narushima, S.; Suda, W.; Imaoka, A.; Setoyama, H.; Nagamori, T.; et al. Th17 cell induction by adhesion of microbes to intestinal epithelial cells. Cell 2015, 163, 367-380. [CrossRef]

46. Sano, T.; Huang, W.; Hall, J.A.; Yang, Y.; Chen, A.; Gavzy, S.J.; Lee, J.Y.; Ziel, J.W.; Miraldi, E.R.; Domingos, A.I.; et al. An il-23r/il-22 circuit regulates epithelial serum amyloid a to promote local effector th17 responses. Cell 2015, 163, 381-393. [CrossRef]

47. Mortha, A.; Chudnovskiy, A.; Hashimoto, D.; Bogunovic, M.; Spencer, S.P.; Belkaid, Y.; Merad, M. Microbiota-dependent crosstalk between macrophages and ilc3 promotes intestinal homeostasis. Science 2014, 343, 1249288. [CrossRef]

48. Celi, P.; Verlhac, V.; Pérez Calvo, E.; Schmeisser, J.; Kluenter, A.-M. Biomarkers of gastrointestinal functionality in animal nutrition and health. Anim. Feed Sci. Technol. 2019, 250, 9-31. [CrossRef]

49. Harris, V.C.; Haak, B.W.; Handley, S.A.; Jiang, B.; Velasquez, D.E.; Hykes, B.L., Jr.; Droit, L.; Berbers, G.A.M.; Kemper, E.M.; van Leeuwen, E.M.M.; et al. Effect of antibiotic-mediated microbiome modulation on rotavirus vaccine immunogenicity: A human, randomized-control proof-of-concept trial. Cell Host Microbe 2018, 24, 197-207. [CrossRef]

50. Lynn, M.A.; Tumes, D.J.; Choo, J.M.; Sribnaia, A.; Blake, S.J.; Leong, L.E.X.; Young, G.P.; Marshall, H.S.; Wesselingh, S.L.; Rogers, G.B.; et al. Early-life antibiotic-driven dysbiosis leads to dysregulated vaccine immune responses in mice. Cell Host Microbe 2018, 23, 653-660. [CrossRef]

51. Luo, Q.; Cui, H.; Peng, X.; Fang, J.; Zuo, Z.; Deng, J.; Liu, J.; Deng, Y. Intestinal iga(+) cell numbers as well as iga, igg, and igm contents correlate with mucosal humoral immunity of broilers during supplementation with high fluorine in the diets. Biol. Trace Elem. Res. 2013, 154, 62-72. [CrossRef] [PubMed]

52. Isaacson, R.; Kim, H.B. The intestinal microbiome of the pig. Anim. Health Res. Rev. 2012, 13, 100-109. [CrossRef] [PubMed]

53. Brunse, A.; Martin, L.; Rasmussen, T.S.; Christensen, L.; Skovsted Cilieborg, M.; Wiese, M.; Khakimov, B.; Pieper, R.; Nielsen, D.S.; Sangild, P.T. Effect of fecal microbiota transplantation route of administration on gut colonization and host response in preterm pigs. ISME J. 2019, 13, 720. [CrossRef]

54. Reetta, S.; Susana, F.; Eero, M.; Jonna, J.; De Vos, W.M.; Perttu, A. Fecal transplantation treatment of antibiotic-induced, noninfectious colitis and long-term microbiota follow-up. Case Rep. Med. 2014, 2014, 913867.

55. O'Toole, P.W.; Cooney, J.C. Probiotic bacteria influence the composition and function of the intestinal microbiota. Interdiscip. Perspect. Infect. Dis. 2008, 2008, 175285. [CrossRef] [PubMed]

56. Liu, C.; Zhang, C.; Lv, W.; Chao, L.; Li, Z.; Shi, D.; Guo, S. Structural modulation of gut microbiota during alleviation of suckling piglets diarrhoea with herbal formula. Evid. Based Complement. Altern. Med. 2017, 2017, 1-11. [CrossRef] [PubMed]

57. Correa, R.O.; Fachi, J.L.; Vieira, A.; Sato, F.T.; Vinolo, M.A.R. Regulation of immune cell function by short-chain fatty acids. Clin. Transl. Immunol. 2016, 5, e73. [CrossRef]

58. Tan, J.; McKenzie, C.; Potamitis, M.; Thorburn, A.N.; Mackay, C.R.; Macia, L. The role of short-chain fatty acids in health and disease. Adv. Immunol. 2014, 121, 91-119.

59. Konstantinov, S.R.; Awati, A.A.; Williams, B.A.; Miller, B.G.; Jones, P.; Stokes, C.R.; Akkermans, A.D.L.; Smidt, H.; De Vos, W.M. Post-natal development of the porcine microbiota composition and activities. Environ. Microbiol. 2006, 8, 1191-1199. [CrossRef] 
60. Daly, K.; Darby, A.C.; Hall, N.; Nau, A.; Bravo, D.; Shirazi-Beechey, S.P. Dietary supplementation with lactose or artificial sweetener enhances swine gut lactobacillus population abundance. Br. J. Nutr. 2014, 111 (Suppl. 1), S30. [CrossRef]

61. Jiao, J.; Wu, J.; Zhou, C.; Tang, S.; Wang, M.; Tan, Z. Composition of ileal bacterial community in grazing goats varies across non-rumination, transition and rumination stages of life. Front. Microbiol. 2016, 7, 1364. [CrossRef] [PubMed]

62. Shang, Q.; Shan, X.; Cai, C.; Hao, J.; Li, G.; Yu, G. Dietary fucoidan modulates the gut microbiota in mice by increasing the abundance of lactobacillus and ruminococcaceae. Food Funct. 2016, 7, 3224. [CrossRef] [PubMed]

63. Vujkovic-Cvijin, I.; Swainson, L.; Chu, S.; Ortiz, A.; Santee, C.; Petriello, A.; Dunham, R.; Fadrosh, D.; Lin, D.; Faruqi, A. Gut-resident lactobacillus abundance associates with ido1 inhibition and th17 dynamics in siv-infected macaques. Cell Rep. 2015, 13, 1589-1597. [CrossRef] [PubMed]

64. Hu, J.; Ma, L.; Nie, Y.; Chen, J.; Zheng, W.; Wang, X.; Xie, C.; Zheng, Z.; Wang, Z.; Yang, T.; et al. A microbiota-derived bacteriocin targets the host to confer diarrhea resistance in early-weaned piglets. Cell Host Microbe 2018, 24, 817-832. [CrossRef] [PubMed]

65. Fukudome, I.; Kobayashi, M.; Dabanaka, K.; Maeda, H.; Okamoto, K.; Okabayashi, T.; Baba, R.; Kumagai, N.; Oba, K.; Fujita, M. Diamine oxidase as a marker of intestinal mucosal injury and the effect of soluble dietary fiber on gastrointestinal tract toxicity after intravenous 5-fluorouracil treatment in rats. Med. Mol. Morphol. 2014, 47, 100-107. [CrossRef] [PubMed]

66. Rokkas, T.; Vaja, S.; Murphy, G.M.; Dowling, R.H. Postheparin plasma diamine oxidase in health and intestinal disease. Gastroenterology 1990, 98, 1493-1501. [CrossRef]

67. Xun, W.; Shi, L.; Zhou, H.; Hou, G.; Cao, T.; Zhao, C. Effects of curcumin on growth performance, jejunal mucosal membrane integrity, morphology and immune status in weaned piglets challenged with enterotoxigenic escherichia coli. Int. Immunopharmacol. 2015, 27, 46-52. [CrossRef]

68. Odamaki, T.; Kato, K.; Sugahara, H. Effect of probiotic yoghurt on animal-based diet-induced change in gut microbiota an open, randomised, parallel-group study. Benef. Microbes 2016, 7, 1-12. [CrossRef]

69. Anderson, J.L.; Edney, R.J.; Whelan, K. Systematic review: Faecal microbiota transplantation in the management of inflammatory bowel disease. Aliment. Pharmacol. Ther. 2012, 36, 503-516. [CrossRef]

70. Cammarota, G.; Ianiro, G.; Gasbarrini, A. Fecal microbiota transplantation for the treatment of clostridium difficile infection: A systematic review. J. Clin. Gastroenterol. 2014, 48, 693. [CrossRef]

71. Quraishi, M.N.; Widlak, M.; Bhala, N.; Moore, D.; Price, M.; Sharma, N.; Iqbal, T.H. Systematic review with meta-analysis: The efficacy of faecal microbiota transplantation for the treatment of recurrent and refractory clostridium difficile infection. Aliment. Pharmacol. Ther. 2017, 46, 479. [CrossRef]

72. Ping, Z.; Bing, Y.; Jun, H.; Gang, T.; Yuheng, L.; Xiangbing, M.; Keying, Z.; Lianqiang, C.; Daiwen, C. Protective effects of dietary arginine supplementation against oxidative stress in weaned piglets. Br. J. Nutr. 2013, 109, 2253-2260.

73. Gresse, R.; Chaucheyras-Durand, F.; Fleury, M.A.; Van de Wiele, T.; Forano, E.; Blanquet-Diot, S. Gut microbiota dysbiosis in postweaning piglets: Understanding the keys to health. Trends Microbiol. 2017, 25, 851-873. [CrossRef] [PubMed]

74. Li, X.; Li, X.; Shang, Q.; Gao, Z.; Hao, F.; Guo, H.; Guo, C. Fecal microbiota transplantation (fmt) could reverse the severity of experimental necrotizing enterocolitis (nec) via oxidative stress modulation. Free Radic. Biol. Med. 2017, 108, 32-43. [CrossRef] [PubMed]

75. Kelly, J.R.; Borre, Y.; Brien, C.O.; Patterson, E.; Aidy, S.E.; Deane, J.; Kennedy, P.J.; Beers, S.; Scott, K.; Moloney, G. Transferring the blues: Depression-associated gut microbiota induces neurobehavioural changes in the rat. J. Psychiatr. Res. 2016, 82, 109-118. [CrossRef] [PubMed]

76. Agata, K.; Lidia, C.; Jerzy, S.; Aleksandra, S.; Katarzyna, K.; Irena, F. Exogenous il-2 controls the balance in th1, th17, and treg cell distribution in patients with progressive rheumatoid arthritis treated with tnf-alpha inhibitors. Inflammation 2015, 38, 765-774.

77. Shameli, A.; Yamanouchi, J.; Tsai, S.; Yang, Y.; Clemente-Casares, X.; Moore, A.; Serra, P.; Santamaria, P. Il-2 promotes the function of memory-like autoregulatory $\mathrm{cd} 8+\mathrm{t}$ cells but suppresses their development via foxp3+ treg cells. Eur. J. Immunol. 2013, 43, 394-403. [CrossRef] 
78. Pang, X.; Hua, X.; Yang, Q.; Ding, D.; Che, C.; Cui, L.; Jia, W.; Bucheli, P.; Zhao, L. Inter-species transplantation of gut microbiota from human to pigs. ISME J. 2007, 1, 156-162. [CrossRef]

79. Ward, T.; Larson, J.; Meulemans, J.; Hillmann, B.; Lynch, J.; Sidiropoulos, D.; Spear, J.; Caporaso, G.; Ran, B.; Knight, R. Bugbase predicts organism level microbiome phenotypes. BioRxiv 2017, 133462. [CrossRef] 